\title{
RETROALIMENTACIÓN ANTE VARIACIONES EN LA PROBABILIDAD DEL DESEMPEÑO EFECTIVO: UN ESTUDIO DE SUS EFECTOS
}

\author{
Héctor Octavio Silva-Victoria, Jorge Guerrero-Barrios, Andrés Francisco Vargas-Avila \\ y Edgar Rocha-Hernández \\ Facultad de Estudios Superiores Iztacala, UNAM \\ México
}

\begin{abstract}
RESUMEN
Se evaluó el efecto de variar la probabilidad de responder acertadamente ante la retroalimentación del desempeño de estudiantes en tareas de igualación a la muestra de segundo orden (IMSO). Se utilizaron términos metodológicos de la práctica científica en Psicología para diseñar las relaciones que debían establecerse entre dichos términos y así poder evaluar el desempeño posterior en la prueba de transferencia (PT). Participaron 20 estudiantes universitarios asignados a cuatro grupos, en los cuales se varió la probabilidad de responder acertadamente: Grupo $1(p=1.0)$, Grupo $2(p=0.5)$, Grupo $3(p=0.25)$ y Grupo 4 ( $p=0.0)$. Todos los grupos recibieron retroalimentación de sus respuestas durante el entrenamiento. En la PT se homologó la probabilidad de responder de manera acertada y no hubo retroalimentación. Los resultados muestran que los grupos 3 y 4 obtuvieron un desempeño similar al obtenido por los grupos 1 y 2 . Esto sugiere que el aumento a la probabilidad de responder equivocadamente, aunado a la retroalimentación, promueve el desempeño efectivo posterior, y en consecuencia el aprendizaje de la tarea.
\end{abstract}

Palabras Clave:

retroalimentación, variaciones paramétricas, términos metodológicos, igualación a la muestra de segundo orden, aprendizaje

\section{FEEDBACK TO VARIATIONS IN THE PROBABILITY OF EFFECTIVE PERFORMANCE: A STUDY OF ITS EFFECTS}

\begin{abstract}
The effects of varying the probability of effective responses and students' performance feedback in Second - Order Matching to Sample Tasks were evaluated. Methodologic terms of the scientific practice in psychology were used to design the relations to be established between those terms, hence be able to evaluate the later performance in the Transfer Tests. This investigation was formed by twenty college students, assigned to four groups in which was varied the probability of effective responses, being as follows; Group $1(p=1.0)$, Group $2(p=0.5)$, Group $3(p=0.25)$ and Group $4(p=0.0)$. All groups got feed-back of their responses during the Training. In the Transfer Test the probability of responding correctly was homologated and there was not feedback. The results show that the groups 3 and 4 got a similar performance to the one gotten by the groups 1 and 2 . This suggests that the increasing of the probability of getting feedback to wrong responses promotes the later effectively performance, and by consequence, the learning of the task.
\end{abstract}

Keywords:

feedback, probability of effective performance, methodological terms, second - order matching to sample task, learning

Bitácora del Artículo:

| Recibido: 2 de Febrero de 2016 | Aceptado: 2 de Marzo de 2017 | Publicado en línea: Julio-Diciembre de 2016 | 


\title{
RETROALIMENTACIÓN ANTE VARIACIONES EN LA PROBABILIDAD DEL DESEMPEÑO EFECTIVO: UN ESTUDIO DE SUS EFECTOS
}

\author{
Héctor Octavio Silva-Victoria, Jorge Guerrero-Barrios, Andrés Francisco Vargas-Avila \\ y Edgar Rocha-Hernández
}

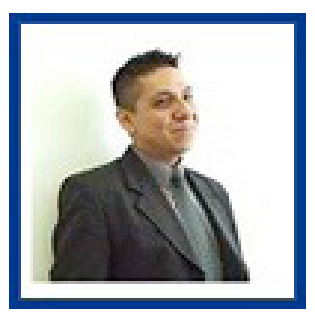

Héctor Octavio Silva-Victoria FES Iztacala UNAM

Correo: silva.unam@gmail.com

Doctor en Psicología en el campo de la educación y el desarrollo por la UNAM. Es profesor de carrera asociado "C" de tiempo completo de la FES Iztacala en el área de Psicología Experimental Humana.

Ver más...

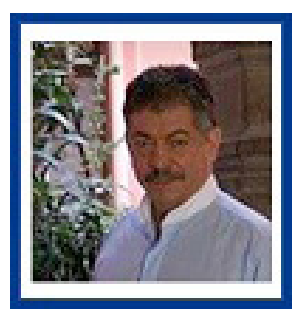

Jorge Guerrero-Barrios

FES Iztacala

Correo: jorgegbarrios@yahoo.com

El doctor Jorge Guerrero es doctor en Psicología. Es profesor de carrera asociado "C" de tiempo completo de la FES Iztacala.

Ver más...

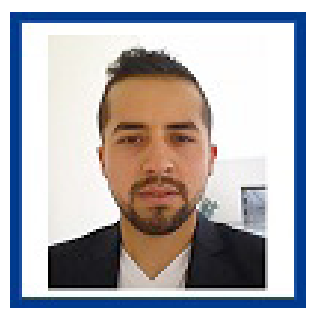

Andrés Francisco Vargas-Ávila

FES Iztacala UNAM

Correo: andresv.unam@gmail.com

Licenciado en Psicología por la Facultad de Estudios Superiores Iztacala, de la Universidad Nacional Autónoma de México. Es Profesor de Asignatura "A" de la Carrera de Psicología de la FES Iztacala de la UNAM, en el Área de Psicología Experimental Animal.

Ver más...

\section{CONTRIBUCIÓN DE LOS AUTORES}

El primer autor coordinó cada una de las etapas de la elaboración de la investigación. Participó en la interpretación de los datos, así como de su representación gráfica y la discusión. | El doctor Jorge Guerrero asesoró en el planteamiento del problema de investigación, el diseño de investigación y en la elección del tipo de métodos estadísticos para la interpretación de los datos. | El lic. Andrés Vargas elaboró parte del material digital, participó en la elección de las relaciones a entrenar y en las relaciones de la prueba de transferencia. | El ultimo autor elaboró el programa en que se utilizó para la investigación. Participó en el diseño de la investigación.

\section{AgRADECIMIENTOS}

Investigación realizada en el marco del Programa de Apoyo a los Profesores de Carrera para Promover Grupos de Investigación PAPCA con clave FES-DIP-PAPCA-2014-60 de la Facultad de Estudios Superiores Iztacala de la Universidad Nacional Autónoma de México.

\section{Datos de Filiación de los Autores}

Facultad de Estudios Superiores Iztacala, UNAM.

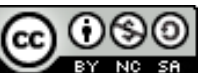

Copyright: (c) 2016 Silva, H.O., Guerrero, J., Vargas A. F., \& Rocha, E.

Este es un artículo de acceso abierto distribuido bajo los términos de la licencia Creative Commons Reconocimiento-NoComercial -Compartirlgual 4.0 Internacional, por lo que su contenido gráfico y escrito se puede compartir, copiar y redistribuir total o parcialmente sin necesidad de permiso expreso de sus autoras con la única condición de que no se puede usar con fines directamente comerciales y los términos legales de cualquier trabajo derivado deben ser los mismos que se expresan en la presente declaración. La única condición es que se cite la fuente con referencia a la Revista Digital Internacional de Psicología y Ciencia Social y a sus autores. 


\section{TABLA DE CONTENIDO}

INTRODUCCIÓN

MÉTOdo

Participantes, 5

Materiales y escenario, 5

Tarea, 5

Diseño experimental, 6

Procedimiento, 6

Mediciones, 7

Análisis estadísticos, 7

Resultados

Discusión

CONCLUSIONES 


\section{INTRODUCCIÓN}

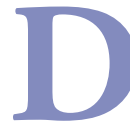
entro del marco de la investigación cuantitativa en Psicología, surgió un procedimiento que permite manipular las variables de interés para el investigador, la discriminación condicional.

Autores como Trigo y Martínez (1994: 69) describen este procedimiento por sus implicaciones experimentales, destacando la relación de condicionalidad entre las respuestas discriminativas y mencionando las tareas de igualación a la muestra como representaciones operativas de dicho arreglo de contingencias.

Por su parte, Ribes et al. (2004) coinciden en que el procedimiento de igualación de la muestra ha sido empleado para el análisis sistemático de la discriminación condicional, además de que dicho procedimiento se ha caracterizado por su alto grado de flexibilidad cuando se ajusta a los diferentes objetivos de las investigaciones donde se emplea. De acuerdo con lo anterior, Valero y Luciano (1997: 600) afirman:

Este procedimiento se ha aplicado al estudio de discriminaciones múltiples entre estímulos visuales y auditivos, relaciones entre números, conceptos verbales abstractos, creación de intraverbales en lenguaje, tareas de lectura, el control estimular en el uso de drogas o en problemas como la agrafia, etc., en personas con y sin retraso. En Valero y Luciano (1992a) puede encontrarse una revisión exhaustiva de los diversos conceptos, procedimientos y estudios realizados sobre el tema hasta la fecha.

Además debe considerarse el objetivo por el cual este tipo de procedimiento resulta útil para los investigadores; Trigo y Martínez (1994: 69-70) lo plantean en términos generales como una herramienta de búsqueda, con la cual se pretende explorar la diversidad de las características de los procesos discriminativos complejos y así poder evaluar la influencia de variables específicas que afectan estos procesos.

Uno de los parámetros explorado con este tipo de procedimientos es el relacionado con las variaciones en la probabilidad de ocurrencia de algún evento. Se pueden mencionar principalmente estudios relacionados con la variación de la probabilidad de reforzamiento. Al respecto, Flores y Bruner (2007) realizaron un estudio donde se evaluaron los efectos de incrementar la duración del intervalo entre reforzadores mediante la reducción de la probabilidad de reforzamiento en la precisión de palomas en tareas de igualación a la muestra, encontrando que la velocidad de adquisición fue más rápida y el índice de discriminación más alto para las palomas expuestas al intervalo más largo entre reforzadores. Además acotan que "en algunos estudios se ha reportado que la variación en la probabilidad de reforzamiento mejora la precisión del responder (e.g., De Long \& Wasserman, 1981; Santi \& Roberts, 1985a, 1985b; Urcuioli, 1990a, 1990b)" (Flores y Bruner, 2007: 33).

Por otra parte, diversos estudios (Carpio et al., 1986; Carpio et al.,1989; Ribes y Torres, 1996; Ribes et al., 1999; Torres et al., 2003) se han desarrollado sobre la probabilidad de reforzamiento utilizando programas definidos temporalmente (o ciclos T) interesados en la correlación entre la tasa de respuestas, y precisión de las mismas, con las variaciones en la probabilidad de reforzamiento.

En los estudios revisados se manipula la probabilidad de ocurrencia de un evento que es contingente de otro, el reforzamiento, es decir, un evento posterior a la ocurrencia de una respuesta específica. Sin embargo no se encontraron antecedentes de la manipulación de la probabilidad de ocurrencia del evento anterior, esto es, responder efectivamente. Cabe mencionar que los estudios realizados a la fecha se han efectuado utilizando sujetos experimentales infrahumanos y con el procedimiento de igualación a la muestra.

En la presente investigación se manipuló la probabilidad de responder efectivamente en tareas de igualación a la muestra de segundo orden; para lograrlo era imprescindible incluir la retroalimentación, ya que mediante ésta se enfatizaba si el desempeño de los participantes, durante el entrenamiento, era efectivo o inefectivo.

Antes de revisar algunos estudios donde la variable independiente es la retroalimentación, es importante matizar que ésta consiste en poner en contacto a un individuo con las características morfológicas y funcionales de su propio desempeño ante una tarea, prueba o problema en un momento posterior a dicho desempeño. De acuerdo con Silva et al. (2014: 39):

Implica la descripción de lo que el individuo hizo, para qué lo hizo y en qué situación lo hizo, si se cumplió o no el criterio a satisfacer, e incluso mencionar las posibles variantes del desempeño del mismo que pudieron haber dado lugar al cumplimiento de criterios didácticos (v.gr., el caso de haber errado o haberse desempeñado de manera inefectiva) o las variantes de su desempeño que pudieron haber sido consideradas como inefectivas.

Esta variable es susceptible de ser manipulada de distintas maneras, según se evidencia en trabajos como el de Carpio et al. (2003), quienes evaluaron el efecto de variar el tipo de retroalimentación en el desempe- 
ño efectivo de universitarios en tareas de identificación de términos metodológicos de Psicología Experimental, concluyendo que el tipo de retroalimentación afecta la ejecución y la precisión del desempeño en tareas posteriores, siendo los tipos de retroalimentación extrasituacional y transituacional los más efectivos para ello.

En un estudio anterior, Ribes y Martínez (1990) variaron la demora de retroalimentación en la ejecución de estudiantes universitarios, encontrando que la retroalimentación inmediata favorece el desempeño efectivo durante el entrenamiento, mientras que la retroalimentación demorada también favorece, pero en este caso — durante los ensayos de prueba - afirmando que la retroalimentación no sólo afecta la ejecución, sino también la precisión del responder.

Moreno et al. (1994) evaluaron el efecto del entrenamiento observacional y ambos tipos de retroalimentación (demorada e inmediata) sobre el responder verbal e instrumental en tareas de igualación a la muestra, encontrando que al retroalimentar de manera demorada la ejecución verbal es mayor, mientras que al ser la retroalimentación inmediata la ejecución instrumental es más efectiva.

En la indagación de la conducta gobernada por reglas, autores como Cepeda et al. (2008) evaluaron el efecto de las descripciones verbales (presencia o ausencia de textos a completar al final de las sesiones) y la presentación de diferentes densidades de retroalimentación (continua o intermitente) sobre las condiciones de adquisición y transferencia, tanto en el desempeño efectivo en tareas de igualación a la muestra de primer orden, como en la formulación de reglas posterior. Los resultados sugieren: 1) que el desempeño efectivo no necesariamente supone la generación de reglas precisas; 2) que otorgar retroalimentación continua favorece la velocidad de adquisición, es decir, la actualización del desempeño efectivo en tareas de esta índole, y 3) que la presentación de textos incompletos desempeña el papel de descripción instruccional, y por tal tiene efecto positivo en términos de adquisición de respuestas.

Los autores mencionados (Moreno et al., 1994; Carpio et al., 2003; Cepeda et al., 2008) comparten un supuesto descrito inicialmente por Ribes y Martínez (1990): que la retroalimentación por sí misma no supone una condición suficiente de promoción del comportamiento variado y efectivo —inteligente-(Ribes, 1990), sino que también desempeñan un papel importante las características de la situación o tarea (como factores disposicionales y situacionales) y los propios criterios de ajuste a satisfacer en la interacción.

Considerando la importancia de dichos factores que afectan tanto la dificultad de la tarea $-y$ por tal el desempeño en la misma- como las características de la retroalimentación -la cual va en función del desempeño del participante-, el presente estudio pretende abonar evidencia a favor de las variaciones paramétricas en las características de la tarea a realizar.

La propuesta que se presenta consiste en manipular la probabilidad de desempeñarse efectivamente ante la retroalimentación, teniendo en cuenta que dichas variaciones no sólo influyen en términos de la retroalimentación, sino también en las circunstancias bajo las cuales ésta es posible respecto a los criterios de la tarea a satisfacer. De tal forma el propósito fue evaluar el efecto de variar las probabilidades de responder de manera efectiva en tareas de igualación a la muestra de segundo orden (IMSO) ante la retroalimentación usando términos metodológicos de la Psicología.

\section{MÉTodo}

\section{Participantes}

Fueron 20 estudiantes universitarios de la carrera de Psicología que participaron en el desarrollo del presente estudio; las edades de los participantes variaron entre 19 y 23 años; fueron separados en cuatro grupos, conformados por cinco participantes cada uno. Ninguno de ellos tenía experiencia en la solución de tareas de IMSO. A cambio de su participación recibieron libros relacionados con sus materias, principalmente en las áreas de Sociales y Métodos Cuantitativos.

\section{Materiales y escenario}

El estudio se realizó en una sola sesión de casi 40 minutos en un cubículo de la Unidad de Investigación Interdisciplinaria en Ciencias de la Salud y la Educación (UIICSE), dentro de la FES Iztacala. Las condiciones del cubículo fueron similares a las condiciones de laboratorio, con la diferencia de que se equipó para poderse llevar a cabo sesiones con cuatro participantes simultáneamente. La tarea y el registro de respuestas fue programada en SuperLab (versión 4.0). Las respuestas se efectuaron tanto con el teclado como con el ratón. Los datos fueron registrados y analizados utilizando el programa Statistica.

\section{Tarea}

Se utilizaron tareas de IMSO en cada una de las fases. Se usó un diseño AB constituido por una fase de entrenamiento (A) y una de evaluación o PT (B). Las pruebas consistieron en la presentación de dos estímulos selectores; - uno muestra y cuatro comparativos en cada arreglo-, presentándose retroalimentación en 
cada uno de los ensayos de entrenamiento (según el desempeño), pero sin retroalimentación en la PT.

\section{Diseño experimental}

A cada uno de los grupos se asignó una condición experimental distinta durante la fase de entrenamiento. Los grupos - cuya diferencia radicaba en la variación de la probabilidad de desempeño efectivo durante la fase de entrenamiento (probabilidades de 0.0, 0.25, 0.5 y 1.0)— trabajaron con tareas de IMSO, entrenándose tres tipos de relaciones durante esta fase (sinonimia, inclusividad y singularidad), mientras que para la fase de evaluación se utilizaron las mismas relaciones y tres más (incompatibilidad, secuencia y pertenencia) —relaciones no entrenadas - igualando la probabilidad de desempeño efectivo a 0.25.

\section{Tabla 1}

Condiciones experimentales a las que fueron expuestos los participantes en fases de entrenamiento y evaluación o prueba de transferencia.

TAREA DE IGUALACIÓN A LA MUESTRA DE SEGUNDO ORDEN (IMSO) CON TÉRMINOS METODOLÓGICOS

\begin{tabular}{|c|c|c|c|c|}
\hline \multirow{2}{*}{ Grupos } & Entrenamiento & \multicolumn{3}{|c|}{$\begin{array}{l}\text { Prueba de } \\
\text { transferencia }\end{array}$} \\
\hline & $\begin{array}{l}\text { Tipo de } \\
\text { relación }\end{array}$ & Probabilidad & Tipo de relación & Probabilidad \\
\hline G1 & \multirow{4}{*}{$\begin{array}{l}\text { Sinonimia, } \\
\text { inclusividad y } \\
\text { singularidad }\end{array}$} & 1.0 & \multirow{4}{*}{$\begin{array}{c}\text { Sinonimia, } \\
\text { inclusividad, } \\
\text { singularidad, } \\
\text { incompatibilidad, } \\
\text { secuencia y } \\
\text { pertenencia }\end{array}$} & \multirow{4}{*}{0.25} \\
\hline $\mathrm{G} 2$ & & 0.5 & & \\
\hline G3 & & 0.25 & & \\
\hline G4 & & 0.0 & & \\
\hline
\end{tabular}

\section{Procedimiento}

Se citó a los participantes, mediante el consentimiento informado, en el cubículo en rondas de cuatro y en horarios diferentes para evitar una saturación del espacio disponible. Al inicio se explicaron las instrucciones generales de operación del programa y se les comentó que una vez terminada la tarea se levantaran en silencio y tomaran uno de los libros que estaban sobre un escritorio. Antes de iniciar con la prueba cada participante era asignado a un grupo diferente mediante una clave de usuario que permitía al programa presentar las pruebas correspondientes según el grupo. Al inicio de la prueba se les presentaban las siguientes instrucciones:

Observa los dos estímulos de la parte superior y determina lo que los relaciona (criterio). Posteriormente, usa dicho criterio para determinar cuál de los cuatro estímulos de la parte inferior (comparativos) cumple esa misma relación con el muestra (estímulo de la parte central); dale click al que consideres correcto.
Una vez leídas las instrucciones se pasó directamente a la fase de entrenamiento, tareas de IMSO cuya probabilidad de desempeño efectivo fue variada según el grupo asignado: el grupo 1 tuvo una probabilidad de 1.0, es decir, las cuatro opciones de respuesta (estímulos comparativos) cumplían con el criterio establecido por los estímulos selectores; la probabilidad del grupo 2 fue de 0.5 , esto es, dos de las cuatro opciones de respuesta eran correctas; la probabilidad del grupo 3 fue de 0.25 (probabilidad típica en este tipo de tareas), en donde una de las cuatro opciones de respuesta era la correcta. Para el grupo 4 la probabilidad fue de 0.0, es decir, ninguna de las opciones de respuesta cumplía con el criterio demandado por los estímulos selectores. En la figura 1 se muestra un ejemplo de arreglo en el grupo 4 .

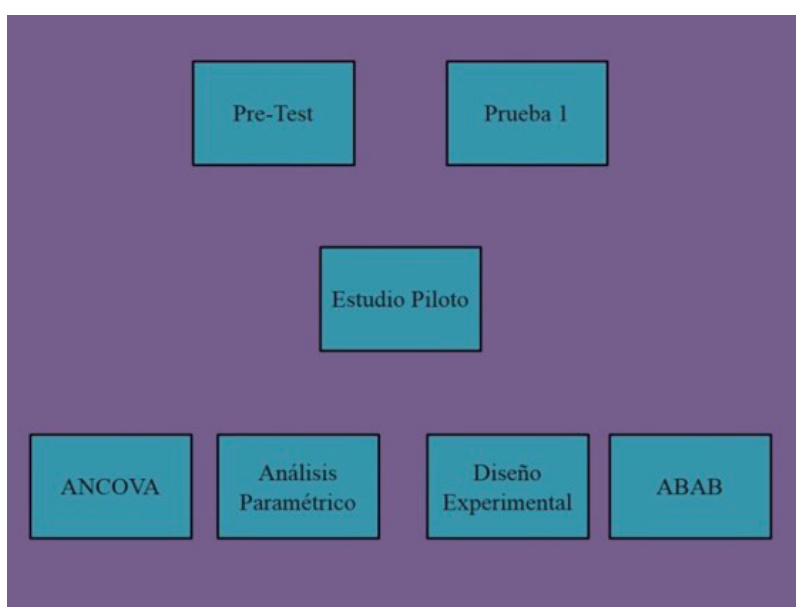

Figura 1

Ejemplo de arreglo en el grupo 4, probabilidad 0.0: ningún estímulo comparativo se ajustaba al criterio.

Los participantes recibieron retroalimentación según su desempeño, y se utilizó el mismo estímulo para todos los grupos en las dos formas: desempeño efectivo e inefectivo. A continuación se muestra el mensaje que fungía como retroalimentación según el desempeño, respectivamente: ¡CORRECTO!

Tu respuesta fue acertada, esa opción cumple con el criterio que existe entre los dos términos superiores.

\section{¡INCORRECTO!}

Tu respuesta no fue acertada, esa opción NO cumple con el criterio que existe entre los dos términos superiores.

En la figura 2 se muestra un ejemplo de arreglo en el grupo 1 (el de probabilidad 1.0) en donde se puede apreciar la característica distintiva de esta condición: todos los estímulos comparativos cumplen con el criterio observado entre los estímulos selectores, es decir, pueden ser usados como sinónimos. 


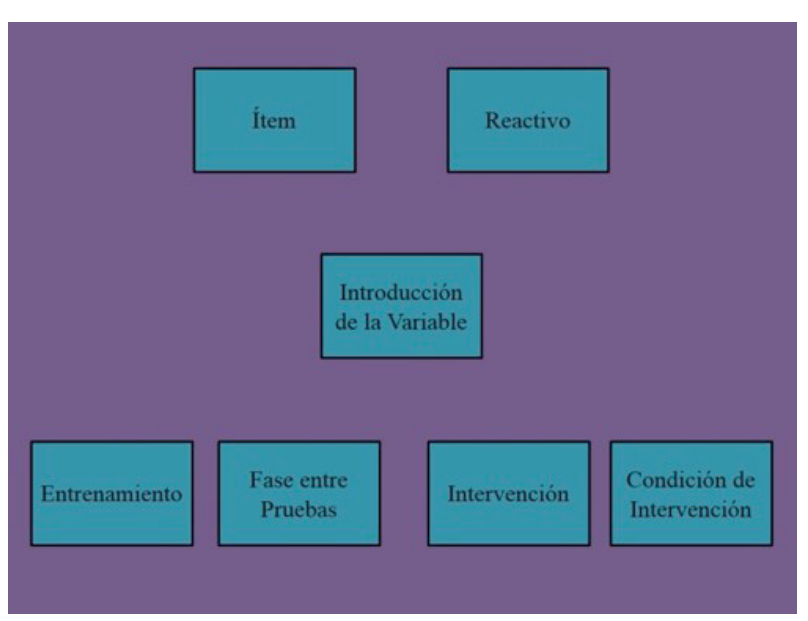

\section{Figura 2}

Ejemplo de arreglo en el grupo 1, probabilidad 1.0: todos los estímulos comparativos cumplen el criterio de equivalencia.

Una vez terminados los 30 ensayos correspondientes a la fase de entrenamiento se inició a la fase de evaluación. La variación de la tarea en la PT en relación con la efectuada durante el entrenamiento fue de tipo extrarrelacional, pues sólo cambiaron las relaciones entre estímulos establecidas durante el entrenamiento, adicionándose tres relaciones. El total de ensayos en esta fase fue de 30, de los cuales 15 fueron con relaciones entrenadas —sinonimia, inclusividad y singularidad - y los otros 15 con relaciones novedosas - incompatibilidad, secuencia y pertenencia-. Las relaciones fueron entendidas de la siguiente manera: 1 ) la relación de sinonimia corresponde a la identificación de los términos con su equivalente; 2) la relación de inclusividad hace referencia a los términos que se incluyen en otros con un carácter más amplio; 3) la relación de singularidad corresponde al establecimiento de semejanzas entre categorías teórica y metodológica; 4) la relación de incompatibilidad refiere a la identificación de términos antónimos; 5) la relación de secuencia corresponde a la identificación de la lógica de presentación de los apartados de un reporte de investigación, y 6) la relación de pertenencia hace referencia a la identificación entre los términos respecto a una categoría que los incluya a ambos.

Antes de iniciar esta fase, se les dio la siguiente instrucción:

Ahora que finalizaste con éxito los ensayos anteriores, y has podido identificar cuando tu desempeño es o no correcto, has de pasar a una prueba final. La dinámica es la misma; da clic en el estímulo comparativo que consideres correcto (de los cuatro que se presentaran en la parte inferior). Antes de empezar, si tienes dudas pregunta a los investigadores.

Una vez terminados los 30 ensayos en la PT se pidió a los participantes que salieran del laboratorio en silencio, no sin antes tomar uno de los libros disponibles en correspondencia por su colaboración.

\section{Mediciones}

La propuesta que se presenta consiste en manipular la probabilidad de desempeñarse efectivamente ante la retroalimentación en tareas de IMSO usando términos metodológicos de la Psicología. Esto se logró modificando los ensayos por grupo. La variable dependiente, correspondiente al desempeño efectivo, correspondió a la contabilización de la cantidad de ensayos resueltos de manera correcta. Las respuestas eran registradas por el programa y recuperadas para su interpretación.

\section{Análisis estadísticos}

Los datos fueron registrados y analizados usando el programa Statistica. Se realizaron dos pruebas de ANOVA de un factor para identificar diferencias significativas entre grupos respecto a sus medias y varianzas, y se aplicó una prueba Tukey para comparar todas las medias para identificar con mayor precisión diferencias entre las mismas.

\section{Resultados}

En la figura 3 se muestra el porcentaje de respuestas correctas en cada una de las fases de acuerdo con el grupo experimental. Se puede apreciar que dicho porcentaje durante el entrenamiento varió en función de la probabilidad de desempeño efectivo en cada grupo, teniendo así al grupo 1 ( $p=1.0$ ) como el que mayores respuestas correctas tuvo, al grupo 2 ( $p=0.5$ ) con $70 \%$, al grupo 3 (grupo de probabilidad típica en tareas de IMSO, $p=0.25$ ) con $33 \%$, y al grupo $4(p=0.0)$, por las características de los arreglos experimentales, con $0 \%$ de respuestas correctas.

Asimismo se puede apreciar que durante la PT en los grupos 1 y 2 el porcentaje de respuestas correctas disminuyó de 100 a $38 \%$ en el grupo 1 y de 70 a $54 \%$ en el grupo 2 , encontrándose resultados contrarios en los grupos 3 y 4 , en donde el porcentaje de respuestas correctas se incrementó de 33 a $49 \%$ y de 0 a $37 \%$, respectivamente.

Se realizaron dos pruebas de ANOVA de un factor. La primera mostró diferencias significativas durante el entrenamiento $[F(3,16)=138.9796 ; p=0.0000]$; una segunda ANOVA, aplicada a la PT, mostró existencia de diferencias significativas entre grupos $[F(3,16)=4.3231 ; p$ $=0.0206]$. Después se aplicó una prueba Tukey a la PT, la cual mostró que los grupos 1 y 4 fueron aquellos con menos diferencia, y que el grupo 2 -respecto a los grupos 1 y 4 - representó la condición de mayor diferencia. 


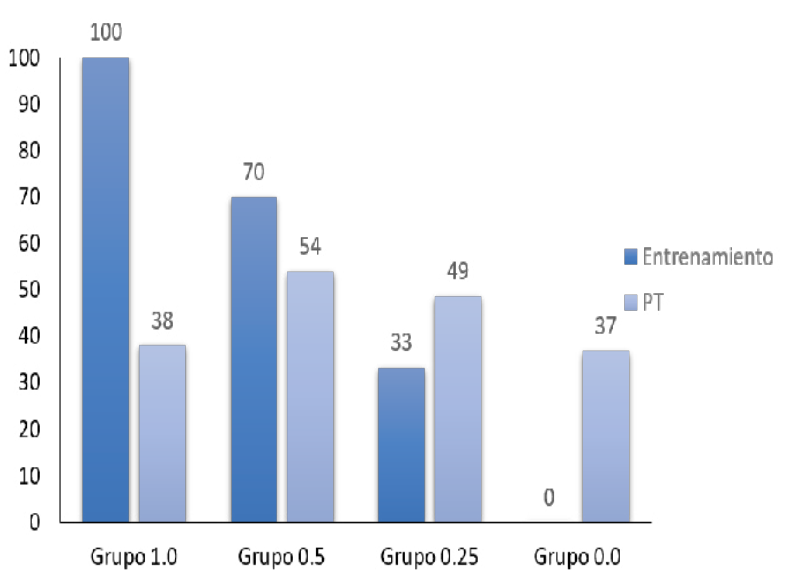

Figura 3

Porcentaje de respuestas correctas por grupos en fase de entrenamiento y en fase de evaluación (PT).

En la figura 4 se aprecia el porcentaje de respuestas correctas en la PT por tipo de relación, es decir, entrenadas o no entrenadas. Obsérvese cómo en todos los grupos (sin importar la condición de entrenamiento) las relaciones no entrenadas tuvieron mayores porcentajes, siendo el grupo 2 (probabilidad 0.5) el que mayor número de respuestas correctas tuvo (37\%), y los grupos 1 y 4 (probabilidad 1.0 y 0.0 , respectivamente) los que menor porcentaje de respuestas correctas obtuvieron tanto en las relaciones no entrenadas como en las entrenadas. Por su parte, el grupo 2 obtuvo el mayor porcentaje en relaciones entrenadas. Al aplicar una ANOVA de un factor a la PT se encontró que existen diferencias significativas en cuanto al desempeño en las relaciones no entrenadas $[F(3,16)=3.7423 ; p=0.0328]$.

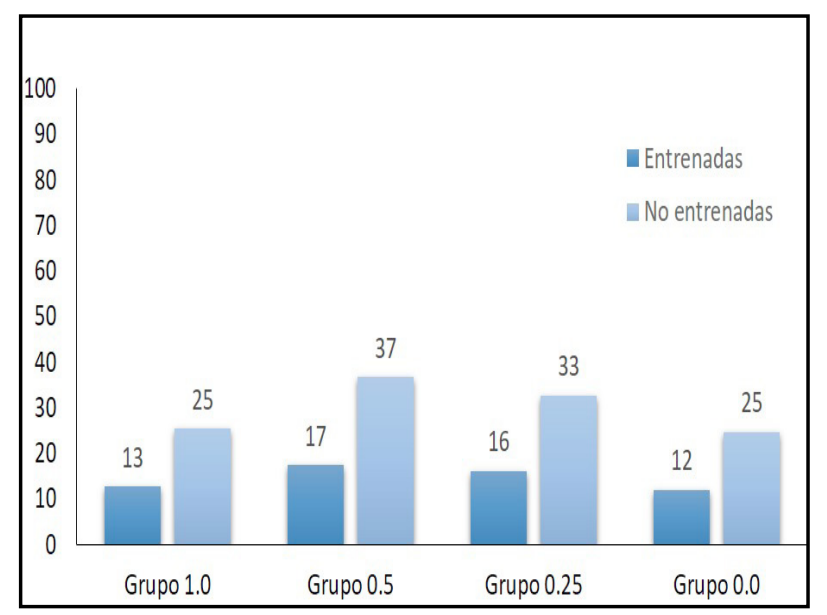

Figura 4

Porcentaje de respuestas correctas por grupo en fase de evaluación por tipo de relación: entrenadas y no entrenadas.
Una prueba de Tukey aplicada posteriormente marcó diferencias entre el grupo 2 y los grupos 1 y 4 (probabilidad de 1.0 y 0.0 , respectivamente). Por su parte, en la prueba de ANOVA aplicada a las relaciones entrenadas no se encontraron diferencias significativas $[F(3,16)=1.5105 ; p=$ $0.2499]$. Es necesario aclarar que ni en las pruebas de transferencia de las relaciones entrenadas ni en las no entrenadas se obtuvieron porcentajes mayores a 50\%, con excepción del porcentaje total en la PT del grupo 2 (0.5), que tuvo $54 \%$, diferencia que no es significativamente diferente al nivel de azar.

\section{Discusión}

Coherentes con el propósito de este estudio: evaluar el efecto de variar la probabilidad de desempeñarse efectivamente ante la retroalimentación en tareas de IMSO, los resultados obtenidos permiten afirmar: 1) que son evidentes los efectos diferenciales de variar la probabilidad de responder de manera efectiva, y 2) que la interacción entre retroalimentación y desempeño es más compleja que el simple efecto sobre la frecuencia de una respuesta; por ejemplo, en el caso del aumento en la precisión de la respuesta, tal como reportan Cepeda et al. (2008) y Ribes y Martínez (1990). Como se pudo observar, las características de las tareas en términos de su dificultad, la probabilidad de desempeñarse de manera efectiva en ellas, así como la retroalimentación de su desempeño, son variables que alteran no sólo la frecuencia en el responder de manera efectiva, sino que permiten alterar la organización funcional total del episodio didáctico con que se pretende entrar en contacto de manera posterior, es decir, afecta la precisión del responder de manera efectiva (Ribes y Martínez, 1990). El aumentar la posibilidad de responder de manera errada o efectiva (grupos 3 y 4), y la retroalimentación de dichos desempeños parece ser una condición que aumenta las probabilidades de desempeñarse de manera efectiva en tareas posteriores.

Este resultado permite suponer que el error debe ser visto como condición necesaria para el aprendizaje y no como impedimento de éste, ya que muchas de las veces —en los escenarios de la vida cotidiana y académica — los individuos se enfrentan a problemas diversos, que pueden ser solucionados de manera particular, pero que fracasan en su intento por solucionarlos, es decir, se equivocan. Además de que en estos escenarios también existen otros individuos que se encargan de enfatizar la equivocación en que se ha incurrido. No obstante, esto genera un tipo de experiencia que posteriormente permite a los individuos enfrentarse a problemas semejantes y solucionarlos. 
Retomando lo anterior (el error como condición necesaria para el aprendizaje), en el marco de la ciencia psicológica esta práctica puede caracterizarse por su asimetría en términos de las habilidades y competencias necesarias para su avance. Este estudio permite afirmar que involucrar a los estudiantes en tareas que impliquen un esfuerzo intelectual mínimo (cualquier respuesta correcta, probabilidad 1.0) o en tareas que impliquen un máximo esfuerzo intelectual (sin posibilidad de respuestas correctas, probabilidad 0.0), y hacerlos entrar en contacto con su desempeño, tienen el mismo efecto en pruebas posteriores, por lo que una tarea que pretenda la promoción de comportamiento variado y efectivo en los estudiantes podría planearse en las condiciones del grupo 2 que, de manera general, fue el grupo que mejores resultados obtuvo. Hace falta más evidencia para poder afirmar un resultado contrario a lo esperado en este estudio: el entrenamiento no fungió como condición de facilitación del desempeño efectivo en pruebas posteriores; antes bien, lo limitó u obstaculizó. Evidencia de ello es el haber sido las relaciones entrenadas durante dicha fase las que, en la PT, tuvieran niveles menores, por debajo de $17 \%$, no siendo dicho porcentaje equiparado al más bajo en las relaciones no entrenadas (25\%). Si bien en éstas el resultado más alto sigue siendo mayor en el grupo 2 (en términos de porcentajes), la diferencia en relación con los obtenidos por otros grupos no es significativa.

\section{Conclusiones}

Se menciona la modalidad de los estímulos usados en estas tareas como posible variable que afectó de manera radical los resultados obtenidos en PT, notando que mientras en este estudio se usaron términos metodológicos de la práctica científica en Psicología, en otros estudios se han usado figuras con colores y formas como propiedad funcionalmente relevante para la igualación (Silva et al., 2005; Guerrero y Ortiz, 2007), propiedades textuales y gramaticales de las palabras empleadas (Varela y Linares, 2002) o estímulos pictóricos (Rodríguez et al., 2011).

Los resultados permiten suponer que el desempeño efectivo de los participantes en el entrenamiento no fue modulado sólo por el tipo de consecuencias a que fueron expuestos, que como se dijo dependía de la probabilidad de responder correctamente. Esto permite fortalecer lo documentado por algunos de los autores citados en la introducción: retroalimentar el desempeño de los estudiantes, por sí mismo, no es causa suficiente para garantizar su mejor desempeño en situaciones futuras, siendo las características de la situación de en- señanza-aprendizaje, y los mismos criterios educativos, elementos relevantes para el ajuste conductual.

Una suposición posterior derivada de las observaciones durante el estudio es que, al parecer, el valor funcional de las consecuencias se define también por lo que el participante se dice acerca de sus aciertos o errores. Esta última suposición permitiría entender cómo los participantes del grupo en que no hubo una consecuencia positiva - porque la probabilidad de responder correctamente fue nula- se desempeñaron con cierta efectividad en la prueba de transferencia. Aunque el equipo de investigación recogió testimonios que fortalecen esta suposición, su registro no fue sistemático, circunstancia que podría alentar investigaciones en esta dirección: estudios sobre la posibilidad de aprender con base en los errores y no en los aciertos. Además, se propone el estudio de la variación paramétrica de las probabilidades de retroalimentar el desempeño ante tareas de IMSO con diferentes estímulos, así como su estudio en tareas diferentes a las aquí usadas.

\section{Referencias}

Carpio, C., Gonzáles, R. \& Ribes, E. (1986). Probabilidad de reforzamiento y su señalización en un programa definido temporalmente. Revista Mexicana de Análisis de la Conducta, 12(2), 89-104.

Carpio, C., Pacheco, V., Ambriz, G. \& Ribes, E. (1989). Efectos de la probabilidad y magnitud de reforzamiento en un programa temporal con distintos requisitos de respuesta. Revista Mexicana de Análisis de la Conducta, 15(1), 55-87.

Carpio, C., Pacheco, V., Carranza, N., Flores, C. \& Canales, C. (2003). Tipos de retroalimentación en el aprendizaje de términos metodológicos de la psicología experimental. Anales de psicología, 19(1). Recuperado de

http://www.redalyc.org/articulo.oa?id=16701910

Cepeda, M., López, A., Moreno, D., Plancarte, P., Arroyo, R. \& Hickman, H. (2008). Efectos de las descripciones y el reforzamiento sobre la generación de reglas. Universitas Psychologica, 8(2). Recuperado de

http://www.scielo.org.co/pdf/rups/v8n2/v8n2a06

Flores, C. \& Bruner, C. (2007). Efectos de diferentes probabilidades de reforzamiento sobre la precisión de palomas en tareas de igualación a la muestra: alargando la duración del intervalo entre reforzadores. Revista Mexicana de Análisis de la Conducta, 33(1), 31-41.

Guerrero, A. \& Ortiz, G. (2007). El papel de la retroalimentación y la ausencia o presencia de instrucciones en la elaboración de descripciones en tareas de discriminación condicional. Acta Colombiana de Psicología, 10 (1). Recuperado de http://repository.ucatolica.edu.co:8080/handle/10983/508

Moreno, D., Ribes, E. \& Martínez, C. (1994). Evaluación experimental de la interacción entre el tipo de pruebas de transferencia y la retroalimentación en una tarea de discriminación condicional bajo aprendizaje 
Artículo empírico | Retroalimentación ante variaciones en la probabilidad del desempeño afectivo.... Silva-Victoria y cols.

observacional. Revista Latina de Pensamiento y Lenguaje, 2(2), 245-286.

Ribes, E. (1990). Psicología General. México: Trillas

Ribes, E. \& Martinez, H. (1990). Interaction of Contingencies and Rule Instructions in the Performance of Human Subjects in Conditional Discrimination. The Psychological Record, 40, 565-586.

Ribes, E., Ontiveros, S., Torres, C., Calderón, G., Carvajal, J., Martínez, C. \& Vargas, I. (2004). La igualación de la muestra como selección de los estímulos de segundo orden: Efectos de dos procedimientos. Revista Mexicana de Análisis de la Conducta, 31(1), 1-22.

Ribes, E. \& Torres, C. (1996). Efectos de la variación en la probabilidad de reforzamiento correlacionada con dos estímulos neutros en un programa definido temporalmente. Revista Mexicana de Análisis de la Conducta, 22(1), 41-78.

Ribes, E., Torres, C. \& Piña, J. (1999). Comparación de los efectos de la presencia y ausencia de estímulos diferenciales en dos programas temporales con probabilidades variantes igualadas. Acta Comportamentalia, 7(1), 5-29.

Rodríguez, M., Ribes, E., Valencia, L. \& González, L. (2011). Efecto de un entrenamiento observacional con descripciones en la transferencia extradimensional. Revista Mexicana de Análisis de la Conducta, 37(2), 155-175.

Silva, H., Arroyo, A., Carpio, C., Irigoyen, J. \& Jiménez, M. (2005). Teoría del desarrollo y comportamiento creativo: algunas evidencias experimentales. En Carpio, C. e Irigoyen, J. (coord.), Psicología y Educación. Aportes desde la teoría de la conducta (pp. 51-65). México: UNAM-FESI.

Silva, H., Morales, G., Pacheco, V., Camacho, A., Garduño, H. \& Carpio, C. (2014). Didáctica como conducta: una propuesta para la descripción de las habilidades de enseñanza. Revista Mexicana de Análisis de la Conducta, 40(3), 32-46. Recuperado de

http://rmac-mx.org/didactica-como-conducta-unapropuesta-para-la-descripcion-de-las-habilidades-deensenanzal

Torres, C., Ribes, E. \& Mayoral, A. (2003). Efectos de probabilidades complementarias y contrastadas de reforzamiento con y sin señalización en programas temporales. Acta Comportamentalia, 11(2), 130-149.

Trigo, E. \& Martínez, H. (1994). Diseños y procedimientos de validación en la psicología interconductual: Discriminación condicional y estrategias longitudinales. Revista Mexicana de la Conducta, 20(1), 67-82.

Valero, L., \& Luciano, M. (1997). Discriminación condicional en niños: Los efectos de contingencias diferenciales de reforzamiento/tiempo fuera. Psicothema, 9(3), 599-608. Recuperado de http://www.psicothema.com/pdf/130.pdf

Varela, J. \& Linares, G. (2002). La transferencia basada en la morfología del objeto de estímulo o en las propiedades textuales. Acta Comportamentalia, 10(1), 87-103. Recuperado de http://www.journals.unam.mx/index.php/acom/article/ view/14624 
Artículo empírico | Retroalimentación ante variaciones en la probabilidad del desempeño afectivo.... Silva-Victoria y cols.

\section{Meta-Análisis del Artículo}

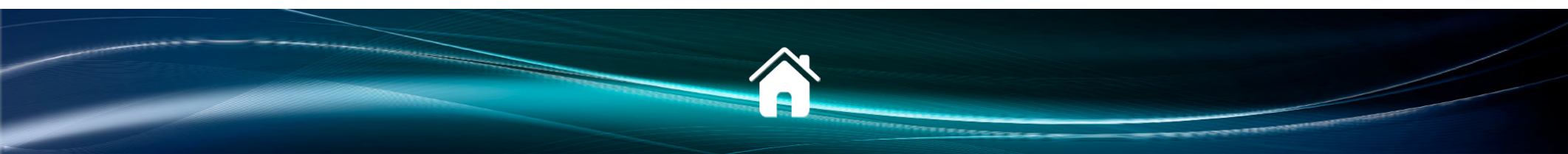


Revista Digital Internacional de Psicología y Ciencia Social |Vol. $2 \mid$ Núm. $2 \mid$ Julio-Diciembre 2016 | ISSN 1405-5802

\section{Dimensión Cuantitativa}

\section{Perfil de Evaluación entre pares}
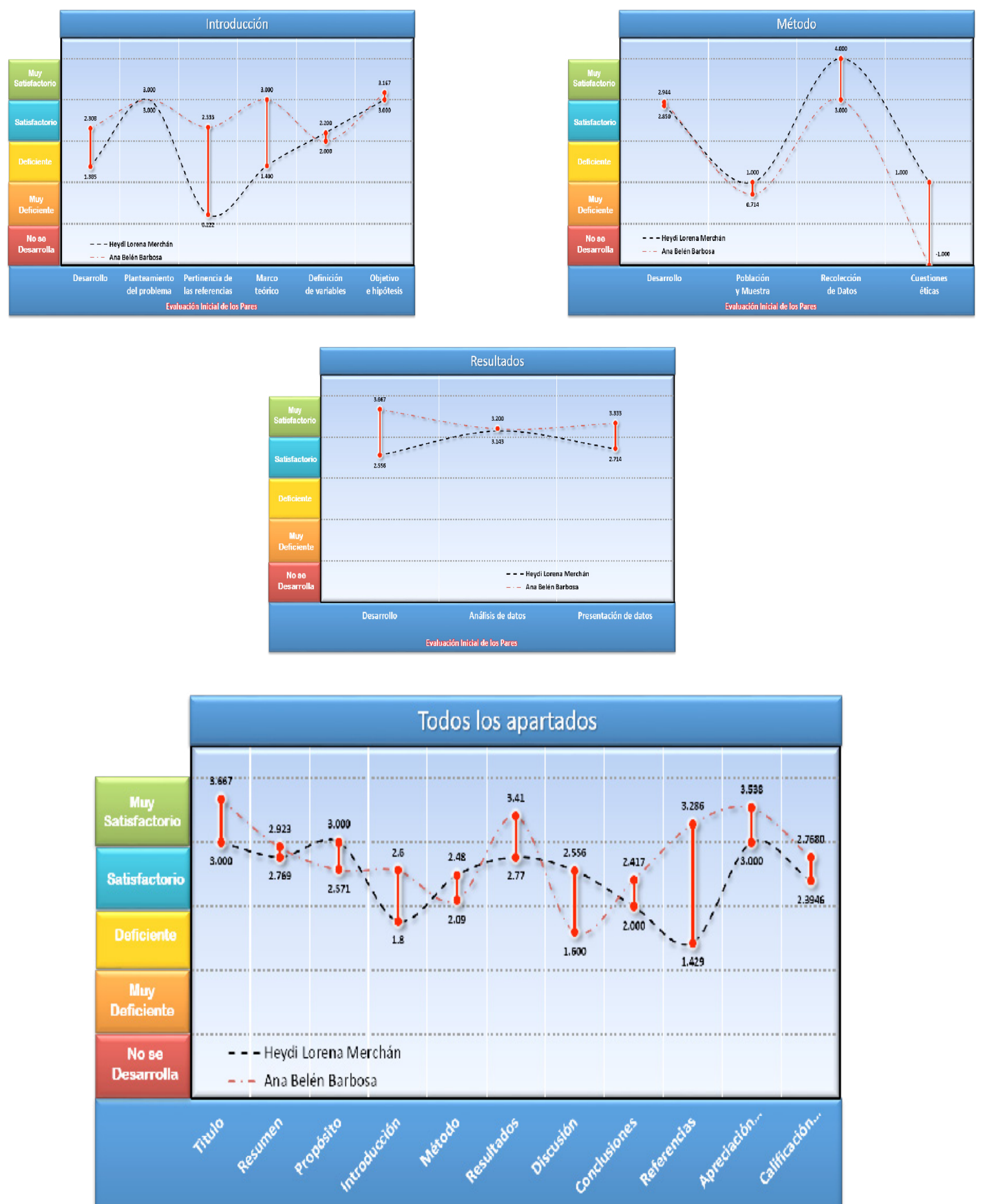

Evaluación Inicial de los Pares 
Artículo empirico | Retroalimentación ante variaciones en la probabilidad del desempeño afectivo.... Silva-Victoria y cols.

\section{Índice de Concordancia}

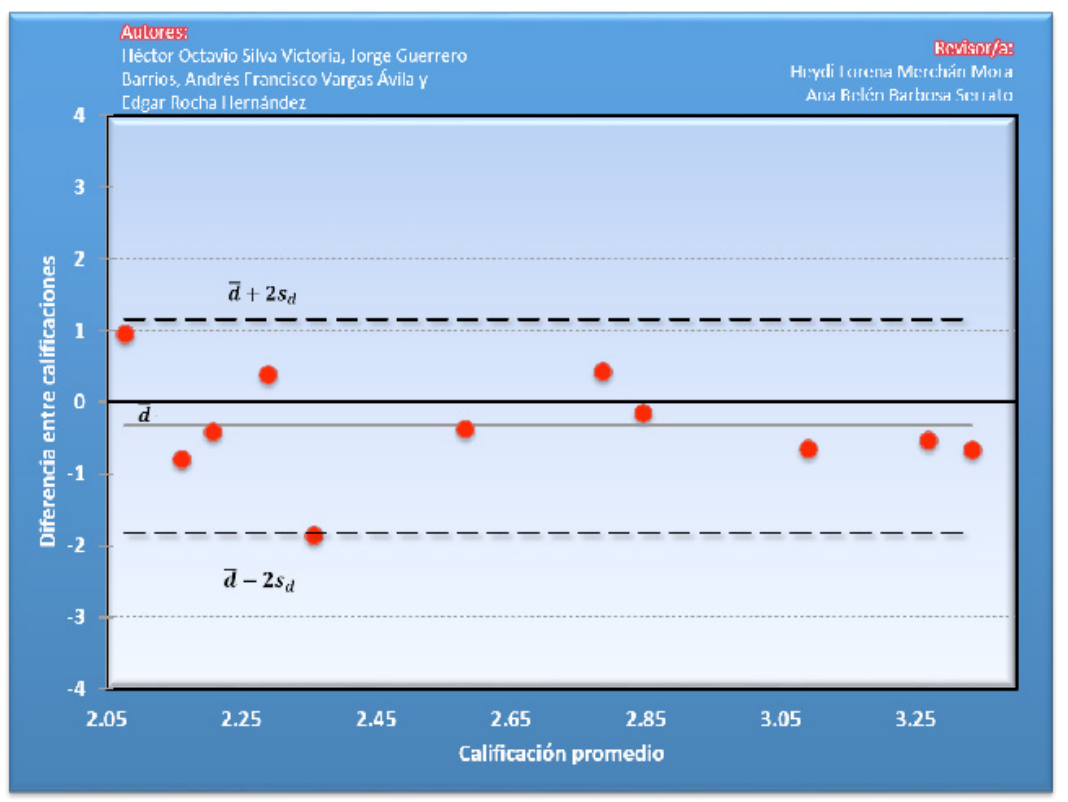

Índice de Acuerdo
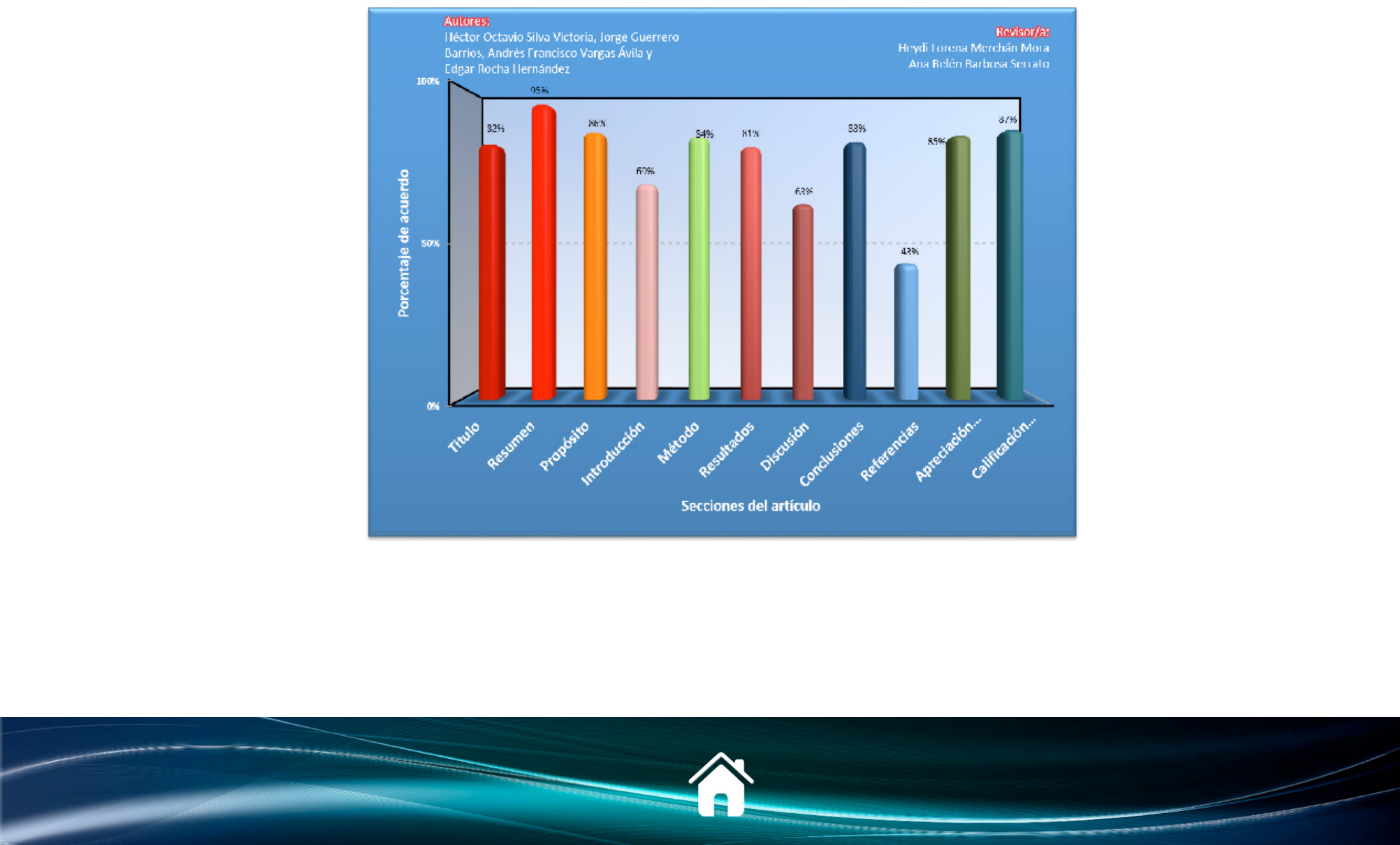


\begin{tabular}{|c|c|}
\hline Revisor 1 & Revisor 2 \\
\hline Heidy Lorena Merchán Moya & Ana Belén Barbosa \\
\hline \multicolumn{2}{|c|}{ Título/Autoría } \\
\hline $\begin{array}{l}\text { Describir en términos del efecto de la variable indepen- } \\
\text { diente desde el inicio del título. }\end{array}$ & $\begin{array}{l}\text { El título es demasiado general, supongo que tiene que } \\
\text { ver con la limitación en el número de palabras. Además } \\
\text { a mi modo de ver, una variable sobra. }\end{array}$ \\
\hline \multicolumn{2}{|c|}{ Resumen } \\
\hline $\begin{array}{l}\text { Cambiar algunas palabras clave, como variaciones pa- } \\
\text { ramétricas y términos metodológicos. Describir los re- } \\
\text { sultados en términos de las diferencias encontradas en } \\
\text { las pruebas estadísticas. }\end{array}$ & $\begin{array}{l}\text { Considero que la conclusión reportada en el resumen } \\
\text { no se deriva de los resultados }\end{array}$ \\
\hline \multicolumn{2}{|c|}{ Próposito del Estudio } \\
\hline $\begin{array}{l}\text { Mejorar la discusión, por lo tanto se deben incluir más } \\
\text { estudios empíricos que sustenten las inferencias hechas } \\
\text { a partir de los resultados encontrados. }\end{array}$ & $\begin{array}{l}\text { El propósito sugiere evaluar el efecto de dos variables } \\
\text { independientes, pero en el trascurso del manuscrito se } \\
\text { evidencia que solo se manipula sistemáticamente una. }\end{array}$ \\
\hline \multicolumn{2}{|c|}{ Introducción } \\
\hline $\begin{array}{l}\text { Mejorar la revisión empírica, así como la teórica, no } \\
\text { se citan estudios en términos empíricos, esto puede } \\
\text { ayudar a justificar de manera mas explícita las varia- } \\
\text { bles manipuladas en el estudio. Mejorar las hipótesis, } \\
\text { hacerlas evidentes en términos de las diferencias entre } \\
\text { los grupos y el entrenamiento manipulado. }\end{array}$ & $\begin{array}{l}\text { Recomiendo revisar nuevamente las variables que pre- } \\
\text { tendían manipular y la contrasten con el procedimien- } \\
\text { to. No hay formulación de hipótesis pero se justifica } \\
\text { por la tradición del área de investigación, se habla mas } \\
\text { bien de resultados esperados. }\end{array}$ \\
\hline \multicolumn{2}{|c|}{ Método } \\
\hline $\begin{array}{l}\text { No se hace explícito si se utilizó o no el consentimiento } \\
\text { informado, incluir. Falta desarrollo en el procedimien- } \\
\text { to, condiciones ambientales de laboratorio, etc, mejo- } \\
\text { rar redacción en este apartado. No se garantiza control } \\
\text { experimental, en la medida que no se hace una linea } \\
\text { de base, la cual pueda fungir como medida de compa- } \\
\text { ración en la etapa de prueba. Se menciona sistemática- } \\
\text { mente una prueba de transferencia sin hacer explicito } \\
\text { cual de tantas es. }\end{array}$ & $\begin{array}{l}\text { No hubo una preprueba, y es importante para cono- } \\
\text { cer el desempeño inicial del participante y/o recono- } \\
\text { cer algún sesgo del participante. El procedimiento no } \\
\text { está completamente desarrollado, no se especifica cla- } \\
\text { ramente en que consiste la tarea en entrenamiento y } \\
\text { prueba de trasferencia. }\end{array}$ \\
\hline
\end{tabular}


Artículo empírico | Retroalimentación ante variaciones en la probabilidad del desempeño afectivo.... Silva-Victoria y cols.

\begin{tabular}{|l|l|}
\hline \multicolumn{1}{|c|}{ Revisor 1 Resultados } \\
\hline $\begin{array}{l}\text { Mejorar las conclusiones de los resultados, esto apo- } \\
\text { yándose con estudios previos a nivel empírico. Justifi- } \\
\text { car el objetivo de los análisis estadísticos. }\end{array}$ & $\begin{array}{l}\text { Las gráficas empleadas para mostrar los resultados son } \\
\text { sencillas y fáciles de comprender. }\end{array}$ \\
\hline \multicolumn{2}{|c|}{ Discusión } \\
\hline $\begin{array}{l}\text { Se recomienda incluir estudios empíricos que permitan } \\
\text { contrastar los resultados encontrados. En varias partes } \\
\text { de este apartado falta citar. }\end{array}$ & $\begin{array}{l}\text { La discusión se puede enriquecer con comparaciones } \\
\text { con estudios antecedentes e implicaciones de los resul- } \\
\text { tados. }\end{array}$ \\
\hline \multicolumn{1}{|c|}{ Conclusiones } \\
\hline $\begin{array}{l}\text { Igual, la discusión sería mas sustanciosa si se incluye } \\
\text { más autores y estudios. }\end{array}$ & $\begin{array}{l}\text { Los resultados no son contundentes para alguna de las } \\
\text { conclusiones que mencionan. }\end{array}$ \\
\hline \multicolumn{2}{|c|}{ Referencias } \\
\hline \begin{tabular}{l} 
Revisar todas las referencias, adecuar el formato APA \\
\hline
\end{tabular} & Complementar los antecedentes del problema a investigar, \\
\hline
\end{tabular}




\section{Historia del Proceso}

\section{EDITORIAL}

